\title{
UNSATURATED SEEPAGE FLOW FROM A HORIZONTAL BOUNDARY*
}

\author{
$\mathrm{By}$ \\ R. A. WOODING \\ D.S.I.R., Wellington, New Zealand
}

Introduction. A knowledge of the time-depth history of infiltration from rainfall or irrigation water into a permeable soil is useful for soil-moisture and ground-water studies and in the analysis of the movement and dispersal of dissolved or suspended materials such as nutrients or pollutants. If these materials are subject to adsorption by soil, the concentration profiles which develop are influenced by the amount and timedistribution of water application. Since the physical system leads to a rather special form of nonlinear transport equation, its properties are reviewed briefly.

(a) Since the soil is generally unsaturated in the infiltration zone, flow is induced by capillarity as well as gravity, and the system exhibits strongly nonlinear properties. Comprehensive reviews $[1,2]$ discuss the literature on this problem.

(b) Capillarity is subject to hysteresis effects, so that at each point in the porous medium, the relationship between capillary pressure and soil moisture is dependent upon previous history. A convenient mathematical description of the hysteresis phenomenon in porous materials does not exist at present [3, 4].

(c) Usually the principal boundary condition-the availability of water at the soil surface expressed as a flux-exhibits time dependence, and may be a variable with 'stochastic' properties. Only in the case of either ponding or zero input can a steady surface condition be expected to prevail.

(d) In unsaturated flow, some water may be 'bound', e.g. by adsorption or by entrapment in dead-end pores [5]. If the amount of bound water depends nonlinearly upon the total water present in unit volume, there will be some influence upon the flow characteristics.

(e) Variation of permeability with depth may be important, and can become extreme in certain hydrophobic ('water-repellent') surface conditions. It is then possible for the wetting front to become unstable [6] and complicated three-dimensional flows can develop.

(f) Infiltration of water into an air-filled porous medium leads to a two-phase flow problem [7]. The buildup of pressure due to entrapped air reduces the rate of infiltration, and instability such as that discussed in (e) can again occur. Some indications of 'fingering', suggestive of instability, have been observed [8].

Several authors $[1,9,10]$ have examined the problem of infiltration into a uniform porous medium when the moisture content at the surface is suddenly increased, e.g. by flooding, so that the boundary moisture condition has a step-function time dependence. Effects of capillary hysteresis were not considered by these authors, since the problem

* Received June 13, 1973; revised version received October 1, 1973. 
involves wetting only. One approach [2] represents gravity as a perturbation upon capillarity, valid for small times, while a second method $[9,10]$ involves an iterative scheme depending partly upon an analytical representation of the 'most important' part of the moisture characteristic-in the range close to saturation-leading to an asymptotic expansion uniformly valid in time.

Other asymptotic procedures are possible in the case of a gravity-dominated system, when capillarity introduces a singular perturbation. This holds for infiltration into fairly permeable soil, where the depth to the water-table is significant. One approach $[7,11]$ uses the Blasius boundary-layer technique, involving the smooth joining of approximate solutions at a chosen point on the moving profile.

This paper introduces a generalization of the latter problem, including an arbitrary time-dependent boundary condition as described in (c), for which case the effects of hysteresis (b) are encountered. It is necessary that the flow be stable, which means that effects (e) and (f) must be assumed small. The possibility of 'bound' water (d) can be taken into account without significant additional complication. In fact, the scope of the analysis becomes more extensive, since it is found to cover also the problem of singlecomponent ion transport with nonlinear adsorption [12].

The problem, which has analogies for nonlinear gas dynamics, is treated by the method of matched asymptotic expansions, and is applicable to waves of large amplitude. In regions of low moisture gradient, the moisture function is represented asymptotically by an outer expansion, the first term of which takes into account the gravity effect only. This term represents a 'kinematic wave' [13], and is also associated with 'BuckleyLeverett theory' [7]. Higher terms of the expansion include the small diffusive effect due to capillarity. In zones of high moisture gradient, however, capillarity and gravity are of comparable magnitude and the outer expansion breaks down. This is analogous to the problem of a 'head shock' moving through a viscous gas [14]. The profile may then be represented by a suitable inner expansion. Particular attention is given to the location and description of the inner region.

Although this paper is not specifically directed towards the hysteresis problem (b), the point is made that some associated difficulties are reduced by the perturbation method. For the present, it will be assumed that an appropriate representation of the capillary potential as a function of changing moisture content can always be chosen.

Unsaturated flow equations. Consider an isothermal porous medium in which the void spaces are partially filled with liquid, the remaining volume being occupied by air and vapour, so that the liquid phase moves under the influence of both gravity and capillary forces. At the very low Reynolds numbers characteristic of seepage flow, it is assumed that Darcy's law, well-established experimentally for saturated media, is applicable in the modified form [15]

$$
\mathrm{Q}=-\frac{k(\theta) g \rho}{\mu} \operatorname{grad}\left\{Z^{*}+p(\theta) / g \rho\right\}
$$

where $\mathbf{Q}$ is the volume flux of liquid per unit area, $Z^{*}$ is the upward vertical coordinate, $g$ is gravity, while $\rho$ and $\mu$ are the density and dynamic viscosity respectively of the liquid. The dimensionless moisture content $\theta$ is defined as the volume of free liquid per unit volume of medium, including pores; its theoretical upper limit cannot exceed the porosity. 
Since the density and dynamic viscosity of the gas are relatively small, gas pressure differences will be treated as negligible; also, to avoid the possibility of gravitational instability, it will be assumed that the gas can "escape" if necessary so that significant pressures are not built up.

Introducing a standard notation, let

$$
K(\theta)=k(\theta) g \rho / \mu, \quad \Psi(\theta)=p(\theta) / g \rho
$$

represent the hydraulic conductivity and capillary potential respectively. Experimentally, as illustrated in Fig. 1(a, b); both $K$ and $\Psi$ are strongly nonlinear, monotonically increasing functions of $\theta$, which may vary through several orders of magnitude $[16,17,18]$. While hysteresis is negligible in the case of $K(\theta), \Psi(\theta)$ exhibits a pronounced hysteresis loop. Attempts to describe this in terms of Néel's independent-domain theory [19, 20, 21] have not been successful, and recent work $[3,4]$ considers interaction between domains. In the present paper, it appears desirable that the quantities $K$ and $\Psi$ be retained as general functions, no approximations being considered in that aspect of the problem.

If the possibility of 'bound' (e.g. adsorbed) water is included, the equation of continuity is

$$
\frac{\partial}{\partial T}\{\theta+\sigma(\theta)\}+\operatorname{div} Q=0
$$

where $T$ is time and $\sigma(\theta)$ is the amount of bound water per unit volume. The curve of $\sigma$ versus $\theta$ will have positive gradient at very low values of $\theta$, but will be expected to tend to a finite limit as $\theta$ increases. Hysteresis effects are likely to be insignificant.

Eliminating $\mathbf{Q}$ between (1) and (3), using (2), gives the transport equation

$$
\frac{\partial}{\partial T}\{\theta+\sigma(\theta)\}-\frac{\partial K(\theta)}{\partial Z^{*}}=\operatorname{div}\left\{K(\theta) \Psi^{\prime}(\theta) \operatorname{grad} \theta\right\}
$$

where ()$^{\prime} \equiv d / d \theta$. The coefficient $K \Psi^{\prime}$ corresponds to a diffusity, but is complicated by the hysteretic properties of $\Psi^{\prime}(\theta)$.

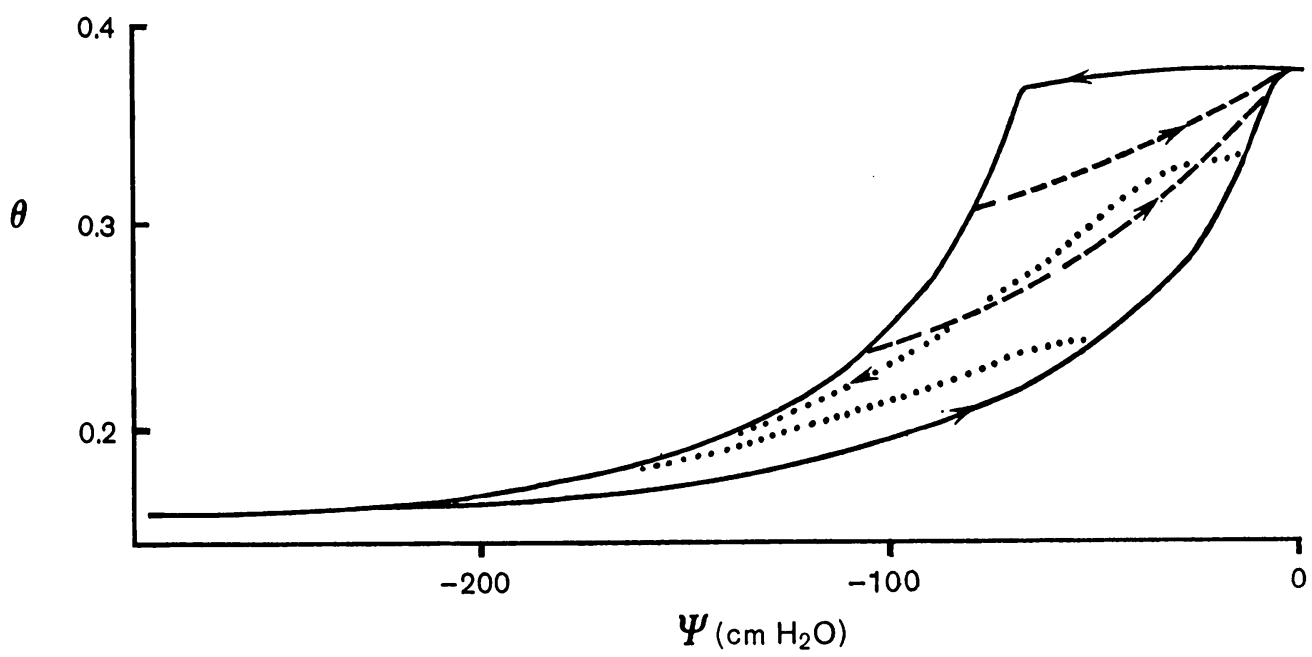

(a) 


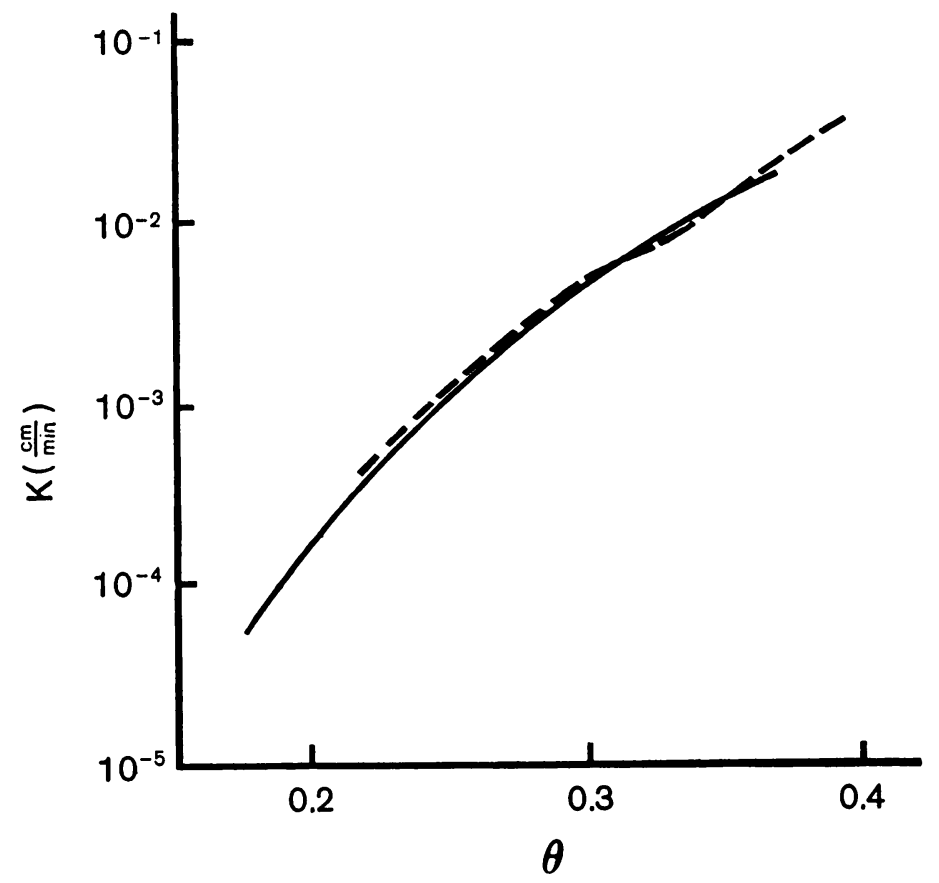

(b)

FIG. 1. Experimental measurements on Rubicon sandy loam by C. G. Topp [17].

(a) Main hysteresis loop (- $\longrightarrow$ in relationship between moisture content $\theta$ and capillary potential $\Psi$. Typical scanning curves are shown for rewetting $(---)$ and redrying $(\cdots \cdots)$.

(b) Relationship between moisture content $\theta$ and hydraulic conductivity $K(\theta)$ showing absence of hysteresis. Rewetting (-); redrying (- - -).

Note that Eq. (4), with $K^{\prime}(\theta)=$ constant and $K \Psi^{\prime}$ replaced by a diffusivity coefficient, corresponds to the transport of an ion component in steady saturated flow with nonlinear adsorption [12]. In that case $\theta$ corresponds to the ion concentration per unit volume of solid medium plus pores, and $\sigma(\theta)$ is the concentration of adsorbed ion.

Flow in one space dimension $\left(Z^{*}\right)$. Let $L$ be an external length of the macroscopic flow path such as porous layer depth or depth to a water-table, and define dimensionless variables

$$
\begin{gathered}
z=Z^{*} / L, t=K_{r}{ }^{\prime} T / L, q=Q / K_{r}{ }^{\prime}, g(\theta)=K(\theta) / K_{r}{ }^{\prime} \\
\kappa(\theta)=K(\theta) \Psi^{\prime}(\theta) /\left(K_{r} \Psi_{r}{ }^{\prime}\right)
\end{gathered}
$$

where $Q$ is the flux in the $Z^{*}$-direction and $K_{r} \equiv K\left(\theta_{r}\right)$, etc. $\theta_{r}$ is a reference value of the moisture content $\theta$, chosen so that the dimensionless coefficients $g(\theta)$, etc., are of order unity in the most important part of the moisture range ( $\theta$ of order unity).

Also, let

$$
b(\theta)=\theta+\sigma(\theta)
$$

represent the total volume of water per unit volume. If 'bound' water is neglected, $\sigma(\theta)=0$. 
In the general form, Eq. (4) becomes

$$
\frac{\partial}{\partial t} b(\theta)-\frac{\partial}{\partial z} g(\theta)=\epsilon \frac{\partial}{\partial z}\left\{\kappa(\theta) \frac{\partial \theta}{\partial z}\right\}
$$

in which the parameter $\epsilon$ is

$$
\epsilon=K_{r} \Psi_{r}^{\prime} / K_{r}^{\prime} L
$$

The magnitude of $\epsilon$ is fairly insensitive to the particular choice of $\theta_{r}$. An assumed approximate $K, \Psi$ relationship [22, 23] of the form

$$
K^{-1} d K / d \Psi=\alpha \text { (constant) }
$$

in (7b) would give $\epsilon=(\alpha L)^{-1}=$ constant. Since (8) appears adequate for some heterogeneous "natural" materials, e.g. loams [24], it follows that (7b) represents a realistic choice of parameter.

Perturbation expansions can be found for either large of small $\epsilon$, the latter case, with which this paper is concerned, being singular.

Infiltration to a water-table. Assume that the soil surface is horizontal, and take the origin of the dimensionless $z$-coordinate (directed upwards) at depth 1 , corresponding to $L$ in the original units, so that $z=0$ is located at the mean position of the water-table. Variations in depth of the water-table will be assumed small in comparison with the scale $L$, i.e. $\xi(t) \ll 1$ (Fig. 2 ).

The surface boundary condition may be expressed as a given time-varying flux or as a time-varying moisture content. For the former, from (1), (2) and (5),

$$
q=-f(t)=-g(\theta)-\epsilon \kappa(\theta) \frac{\partial \theta}{\partial z} \quad(z=1),
$$

where $f(t)$ is a non-negative input function prescribed, for example, from precipitation data. In the present treatment it is necessary that $f(t)$ be slowly-varying so that $f^{\prime}(t) / f(t)$, $f^{\prime \prime}(t) / f(t), \cdots \leq 0(1)$, except at isolated, finite discontinuities in $f$, which are admissible. Initial conditions, which would involve prescribing $\theta$ as a function of $z$ at, say, $t=0$, will not be considered. The properties of the system are such that, if $f(t)$ is prescribed for all $t$, the distribution at $t=0$ is determined by $f(t)$ for a finite range of $t \leq 0$.

At the water-table, the coordinate $z=\xi(t)$ is not known a priori, and depends upon the downward flux $q_{s}(t)$ of ground-water, which must be specified independently. The

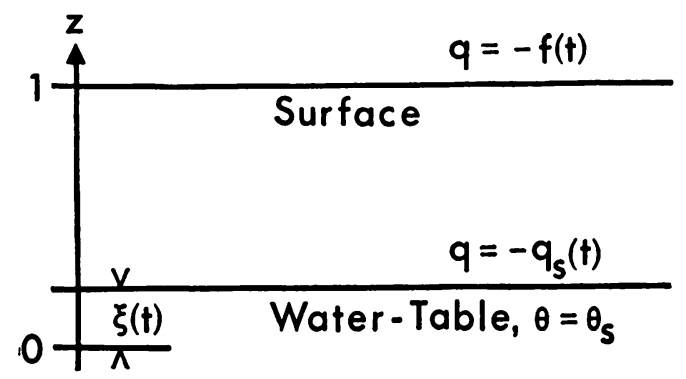

Fig 2. Boundary conditions for unsaturated infiltration through a porous layer to a water-table at $z=\xi(t)$. 
conditions are then

$$
q=-q_{\ominus}(t), \theta=\theta_{\mathrm{s}}(z=\xi(t))
$$

where $\theta_{s}$ is the (constant) saturation value. A further condition is necessary in order to specify $\xi(t)$ uniquely; the fact that the mean value of $\xi(t)$ is assumed known (to specify the position of $z=0$ ) suffices.

Outer expansion. Consider first a regular expansion of $\theta$ in powers of $\epsilon$ :

$$
\theta(z, t)=\theta^{(0)}(z, t)+\epsilon \theta^{(1)}(z, t)+\cdots .
$$

Substitution of (12) into (7) gives

$$
\begin{aligned}
\frac{\partial}{\partial t}\left(b^{(0)}+\epsilon b^{(0) \prime} \theta^{(1)}+\cdots\right)-\frac{\partial}{\partial z}\left(g^{(0)}+\epsilon g^{(0) \prime} \theta^{(1)}\right. & +\cdots) \\
& =\epsilon \frac{\partial}{\partial z}\left\{\left(\kappa^{(0)}+\cdots\right)\left(\frac{\partial \theta^{(0)}}{\partial z}+\cdots\right)\right\}
\end{aligned}
$$

where $b(\theta), g(\theta)$ and $\kappa(\theta)$ have been replaced by their Taylor expansions; the primes signify $d / d \theta^{(0)}, g^{(0) \prime} \equiv g^{\prime}\left(\theta^{(0)}\right)$, etc. Then the coefficients $\theta^{(0)}, \theta^{(1)}, \cdots$ obey the firstorder hyperbolic equations

$$
\begin{gathered}
b^{(0)^{\prime}} \frac{\partial \theta^{(0)}}{\partial t}-g^{(0) \prime} \frac{\partial \theta^{(0)}}{\partial z}=0 \\
\frac{\partial}{\partial t}\left(b^{(0)^{\prime}} \theta^{(1)}\right)-\frac{\partial}{\partial z}\left(g^{(0)^{\prime}} \theta^{(1)}\right)=\frac{\partial}{\partial z}\left\{\kappa^{(0)} \frac{\partial \theta^{(0)}}{\partial z}\right\}
\end{gathered}
$$

with surface boundary conditions (at $z=1$ )

$$
\begin{gathered}
g^{(0)}=f(t) \\
g^{(0)} \theta^{(1)}+\kappa^{(0)} \frac{\partial \theta^{(0)}}{\partial z}=0
\end{gathered}
$$

from (9). Since the order of this system is lower than that of the original one, it is not possible to satisfy the down-stream boundary condition (10). Note that $g(\theta)$ and the coefficients of its Taylor expansion, which depend only upon $K(\theta)$, do not exhibit measurable hysteresis and therefore are regarded as one-valued functions of $\theta . b(\theta)$ is also onevalued.

Eq. (13) is equivalent to

$$
d t /-a^{(0)}=d z / 1=d \theta^{(0)} / 0
$$

where

$$
a^{(0)}=b^{(0) \prime} / g^{(0) \prime}=d b^{(0)} / d g^{(0)} .
$$

Since $\theta^{(0)}$ is constant along any given characteristic, for which the characteristic velocity is $-g^{(0) \prime} / b^{(0) '}$, the characteristics are non-parallel straight lines in the $(z, t)$-plane. The boundary condition (15) combined with (17) gives

$$
g^{(0)}=f\left(\Phi^{(0)}\right)
$$


writing

$$
\Phi^{(0)}=t+a^{(0)}(z-1) .
$$

It is evident from (19) that $\Phi^{(0)}$ can be defined as an inverse function, $f^{-1}\left(g^{(0)}\right)$, and is therefore a function of $\theta^{(0)}$.

From $(20)$, the slope of the $\theta^{(0)}$-profile at $(z, t)$ is

$$
\frac{\partial \theta^{(0)}}{\partial z}=\frac{a^{(0)}}{\Phi^{(0)^{\prime}}-a^{(0)^{\prime}}(z-1)}=\frac{a^{(0)}}{\Phi^{(0)^{\prime}}+\left(t-\Phi^{(0)}\right) a^{(0)^{\prime}} / a^{(0)^{\prime}}}=a^{(0)} / \Delta\left(\theta^{(0)}, t\right) \text { say. }
$$

By means of (21), Eq. (14) can now be written in characteristic form as

$$
\begin{aligned}
\frac{d}{d t}\left(b^{(0)} \theta^{(1)}\right) & =a^{(0)} \frac{\partial}{\partial \theta^{(0)}}\left(\frac{\kappa^{(0)} a^{(0)}}{\Delta}\right) \\
& \left.=\frac{a^{(0)}}{\Delta}\left\{\left(\kappa^{(0)} a^{(0)^{2}} / a^{(0)}\right)^{\prime}-\kappa^{(0)} a^{(0)^{2}} \Phi^{(0)^{\prime}} / a^{(0)^{\prime}}\right)^{\prime} / \Delta\right\}
\end{aligned}
$$

and the boundary condition (16) becomes

$$
b^{(0) \prime} \theta^{(1)}=-\kappa^{(0)} a^{(0) 2} / \Phi^{(0) \prime},
$$

since $\Delta=\Phi^{(0) '}$ at $z=1$. Along a characteristic, each quantity with superscript (0) behaves as a constant. Therefore, integration of (22), subject to (23), gives

$$
\begin{aligned}
b^{(0)^{\prime}} \theta^{(1)}= & \frac{a^{(0)}}{\Delta} \frac{\partial}{\partial \theta^{(0)}}\left\{\left(\kappa^{(0)} a^{(0)^{\prime}} / a^{(0)^{\prime}}\right) \log \left|\Delta / \Phi^{(0)^{\prime}}\right|\right\} \\
= & \frac{a^{(0)}}{\Delta}\left\{\left(\kappa^{(0)} a^{(0)^{2}} / a^{(0)^{\prime}}\right)^{\prime} \log \left|\Delta / \Phi^{(0)^{\prime}}\right|\right. \\
& \left.+\kappa^{(0)} a^{(0)^{\prime}}\left(\Phi^{(0)^{\prime}} / a^{(0)^{\prime}}\right)^{\prime}\left(\Delta^{-1}-\Phi^{(0)^{\prime}-1}\right)-\kappa^{(0)} a^{(0)}\right\} .
\end{aligned}
$$

Eq. (24) exhibits the first influence of capillary hysteresis in the outer expansion, through the coefficient $\kappa^{(0)} \equiv \kappa\left(\theta^{(0)}\right)$, which is seen to be an effect of order $\epsilon$. The spacetime history of $\theta^{(0)}$, which is independent of hysteresis, is known from (19), (20), and so the argument of $\kappa^{(0)}$ is specified. Since the behaviour of an hysteretic function can be determined provided that its previous history is known, $\kappa^{(0)}$ can, in principle, be treated as a known function.

Properties of the outer solution: discontinuities. Except at zones of high gradient in moisture content $\theta$, the principal descriptive features of the infiltration process can be deduced from the leading term of the outer expansion, which is not affected by capillarity.

Following a characteristic $\theta^{(0)}=$ constant, the rate of change of gradient in the moisture content is given approximately by

$$
d / d t\left(\partial \theta^{(0)} / \partial z\right)=-a^{(0)} / \Delta^{2}
$$

from (21). The denominator of (25) is finite except at certain singular points in the $(z, t)$-plane (discussed below). Away from these points, the gradient $\partial \theta^{(0)} / \partial z$ is seen to be increasing with time, irrespective of its sign, provided that $a^{(0)^{\prime}}=\left(b^{(0)^{\prime}} / g^{(0) \prime}\right)^{\prime}<0$; that is, the curve of hydraulic conductivity $K(\theta)$ is concave upwards, which is the usual case. The effect is enhanced if the curve of $b^{(0)}$ versus $\theta^{(0)}$ is concave downwards, which is typical of adsorption processes. With the definition of the $z$-coordinate used here, a 
positive slope (advancing profile) steepens, while a negative slope, or "tail", becomes less steep.

For the advancing profile, the condition $\partial \theta^{(0)} / \partial z>0$ gives $\Delta>0$ from equation (21). However, since $a^{(0)}<0, \Delta$ decreases with time and ultimately changes sign. When the steepening front becomes vertical $(\Delta=0)$, the two conditions

$$
\partial z / \partial \theta^{(0)}=\partial^{2} z / \partial \theta^{(0) 2}=0
$$

must be satisfied simultaneously, giving

$$
\Phi^{(0) \prime} / a^{(0) \prime}=\Phi^{(0) \prime \prime} / a^{(0) \prime}=z-1,
$$

which determines the corresponding values of $z, \theta^{(0)}$ and $t$.

At subsequent times, the solution (19) is multi-valued over a finite range of $\theta^{(0)}$ and $z$, and therefore is not physically tenable. The appropriate one-valued solution possesses a discontinuity, or jump, in the value of $\theta^{(0)}$. Such a discontinuity cannot have physical reality when $\epsilon \neq 0$, since the governing equation (7a) is then parabolic, and the outer solution actually breaks down in that neighbourhood. It is necessary to formulate an inner problem to cover the region in question, while retaining the concept of a discontinuous outer solution which is mathematically convenient.

In this first-order system, the motion of the jump is described by a single conservation condition, and a correction to the jump speed is made at each order of the outer problem. Let the discontinuity be located at $z=\zeta(t)$, where $\zeta(t)$ can be expanded in powers of $\epsilon$ analogous to (11), viz.,

$$
\zeta(t)=\zeta^{(0)}(t)+\epsilon \zeta^{(1)}(t)+\cdots,
$$

and put $\bar{z}=z-\zeta(t)$, so that $\partial /\left.\partial t\right|_{z}=\partial /\left.\partial t\right|_{\bar{z}}-\zeta^{\prime}(t) \partial / \partial \bar{z}$. Here $\zeta^{\prime}(t) \equiv d \zeta / d t$ is the jump velocity. To find an equation involving $\zeta(t)$, let the independent variable $z$ in (7a) be replaced by $\bar{z}$; then

$$
\frac{\partial b}{\partial t}-\left\{\zeta^{\prime}(t) \frac{\partial b}{\partial \bar{z}}+\frac{\partial g}{\partial \bar{z}}\right\}=\epsilon \frac{\partial}{\partial \bar{z}}\left(\kappa \frac{\partial \theta}{\partial \bar{z}}\right)
$$

Now, if the profile of moisture content contains a finite jump from $\theta_{1}(t)$ to $\theta_{2}(t)$ at $z=$ $\zeta(t)$, the result of integrating (29) with respect to $\bar{z}$ through a small range including the origin, and letting that range tend to zero, is

$$
-\zeta^{\prime}(t)[b(\theta)]_{1}^{2}-[g(\theta)]_{1}^{2}=\epsilon\left[\kappa(\theta) \frac{\partial \theta}{\partial \bar{z}}\right]_{1}^{2},
$$

writing $[\theta]_{1}{ }^{2} \equiv \theta_{2}-\theta_{1}$, etc. If $\theta$ is replaced by the outer expansion (11) in (30), and $\zeta^{\prime}(t)$ is expanded following (28), then

$$
\begin{aligned}
& -\zeta^{(0)^{\prime}}(t)\left[b^{(0)}\right]_{1}{ }^{2}=\left[g^{(0)}\right]_{1}{ }^{2}, \\
& -\zeta^{(1)^{\prime}}(t)\left[b^{(0)}\right]_{1}{ }^{2}=\left[\beta^{(0)} \theta^{(1)}\right]_{1}{ }^{2}+\left[\kappa^{(0)} a^{(0)} / \Delta\right]_{1}{ }^{2},
\end{aligned}
$$

where the limits refer to values at the discontinuity, and

$$
\beta^{(0)} \equiv \beta\left(\theta^{(0)}, t\right) ; \beta(\theta, t) \equiv \zeta^{(0)} b^{\prime}(\theta)+g^{\prime}(\theta) .
$$


From (31) and (18) it is readily shown that

$$
1 / a_{1}{ }^{(0)}<-\zeta^{(0) \prime}(t)<1 / a_{2}{ }^{(0)} ;
$$

i.e., the jump speed is intermediate between the characteristic speeds ahead of, and behind, the jump. This follows at once since $-\zeta^{(0)}$ is given by the slope of the chord joining two points on the curve of $g^{(0)}$ versus $b^{(0)}$, whereas $1 / a_{1}{ }^{(0)}, 1 / a_{2}{ }^{(0)}$ are equal to the slopes of the tangents at the two points. (Note that $-\zeta^{(0)}>0$ since flow is in the direction of decreasing $z$.)

When Eq. (20) is applied at both ends of the discontinuity at $z=\zeta(t)$, it follows that

$$
\{1-\zeta(t)\} b_{i}{ }^{(0) \prime}-\left(t-\Phi_{i}{ }^{(0)}\right) g_{i}{ }^{(0) \prime}=0 \quad(i=1,2) .
$$

The results $(35 \mathrm{a}, \mathrm{b})$ respectively are multiplied by $\left(\partial \theta^{(0)} / \partial t\right)_{i}(i=1,2)$ and subtracted from, and added to (30). After expansion using (11) and (28), the $0(1), 0(\epsilon), \cdots$ equations integrate immediately to

$$
\begin{aligned}
\left\{1-\zeta^{(0)}(t)\right\}\left[b^{(0)}\right]_{1}{ }^{2} & =\left[g^{(0)}\right]_{1}{ }^{2} t-\int_{1}^{2} \Phi^{(0)} d g^{(0)} \\
-\zeta^{(1)}(t)\left[b^{(0)}\right]_{1}{ }^{2} & =\left[\left(\kappa^{(0)} a^{(0) 2} / a^{(0)}\right) \log \left|\Delta / \Phi^{(0)^{\prime}}\right|\right]_{1}{ }^{2}
\end{aligned}
$$

Here, use has been made of the result

$$
\begin{aligned}
{\left[\beta^{(0)} \theta^{(1)}\right]_{1}{ }^{2}+\left[\kappa^{(0)} a^{(0)} / \Delta\right]_{1}{ }^{2} } & =\frac{\partial}{\partial t}\left(\int_{1}^{2} \frac{\theta^{(1)} \Delta}{a^{(0)}} d b^{(0)}\right) \\
& =\frac{\partial}{\partial t}\left[\left(\kappa^{(0)} a^{(0)^{2}} / a^{(0)^{\prime}}\right) \log \left|\Delta / \Phi^{(0)^{\prime}}\right|\right]_{1}{ }^{2} .
\end{aligned}
$$

An alternative derivation of (36) and (37), via the conservation integral, is stated in the Appendix.

Elimination of $t$ and $1-\zeta^{(0)}(t)$ between $(35 \mathrm{a}, \mathrm{b})$ and (36) gives a relation between corresponding values of $\theta_{1}{ }^{\left({ }^{(0)}\right.}$ and $\theta_{2}{ }^{(0)}$, which can be written in the alternative forms

$$
\begin{aligned}
& \frac{\left[g^{(0)}\right]_{1}{ }^{2}-\left[b^{(0)} / a^{(0)}\right]_{1}{ }^{2}}{\left[1 / a^{(0)}\right]_{1}{ }^{2}}=\frac{\int_{1}^{2} \Phi^{(0)} d g^{(0)}-\left[\Phi^{(0)} b^{(0)} / a^{(0)}\right]_{1}{ }^{2}}{\left[\Phi^{(0)} / a^{(0)}\right]_{1}{ }^{2}}, \\
& \frac{\left[b^{(0)}\right]_{1}{ }^{2}-\left[a^{(0)} g^{(0)}\right]_{1}{ }^{2}}{\left[a^{(0)}\right]_{1}{ }^{2}}=\frac{\int_{1}{ }^{2} \Phi^{(0)} d g^{(0)}-\left[\Phi^{(0)} g^{(0)}\right]_{1}{ }^{2}}{\left[\Phi^{(0)}\right]_{1}{ }^{2}} .
\end{aligned}
$$

Either can be solved, explicitly or by iteration, in specific cases, and corresponding values of $t$ and $\zeta^{(0)}(t)$ follow by back substitution. The form (39a) is most suitable when $\sigma(\theta)=0,\left(b^{\prime}(\theta)=1\right)$, so that $1 / a^{(0)}=g^{(0) \prime}$, while $(39 \mathrm{~b})$ is preferable when $g^{(0) \prime}=1$, so that $a^{(0)}=b^{(0)}$.

The representations ( $36 \mathrm{ff}$.) apply for a discontinuity which originates within the moisture profile such that $0<\zeta<1$ initially, and the range of integration covers values of $\theta^{(0)}$ for which the outer solution does not exist physically. However, for a finite jump. introduced at the surface $z=1$ by a discontinuity in the function $f(t)$ of $(10)$, different integral representations are needed. For example, the integral in (36) and (39) is replaced 
by the difference of two separate integrals over the ranges $\theta_{i}{ }^{(0)}(1, t)$ to $\theta_{i}{ }^{(0)}\{\zeta(t), t\}$, where $i=1,2$.

Discontinuity at downstream boundary. In addition to the moving discontinuity described in detail above, a second kind of sustained discontinuity can arise in the outer problem. Since the outer expansion satisfies a first-order hyperbolic system, it is not possible to satisfy the downstream boundary condition (10) at the water-table, $z=\xi(t)$, and a "backward-facing" jump appears. (Some linear problems also exhibit this property [25].)

Upstream of (i.e. above) the water-table, the outer expansion (11) is applicable, and may be used to represent $\theta(\xi, t)$. The determination of $\xi(t)$ then parallels that of $\zeta(t)$, putting $\theta_{1}(t)=\theta_{0}, \theta_{2}(t)=\theta(\xi, t), g\left(\theta_{1}\right)=q_{0}(t), \partial \theta /\left.\partial z\right|_{\theta=\theta_{1}}=0$, etc., with appropriate substitutions in the coefficients of the asymptotic expansions. The details of the analysis will be omitted.

Inner expansions. The discontinuities described above are symptoms of the breakdown of the outer representation, and suitable inner expansions must be found to represent the solution in the neighbourhood of such points. For the cases considered here, it is readily shown that the appropriate scale for an inner "stretched" variable is $\epsilon$. In this problem, the inner representation covers the region where the diffusion term in (7) is as significant as the convection terms.

Moving discontinuity. Let the scaled coordinate $Z$ be defined by

$$
Z=\{z-\zeta(t)\} / \epsilon
$$

in $(7 \mathrm{a})$, to give

$$
\epsilon \frac{\partial b}{\partial t}-\left\{\zeta^{\prime}(t) b^{\prime}(\theta)+g^{\prime}(\theta)\right\} \frac{\partial \theta}{\partial Z}=\frac{\partial}{\partial Z}\left\{\kappa(\theta) \frac{\partial \theta}{\partial Z}\right\} .
$$

Here the diffusion term retains its full importance, and the inner problem exhibits quasisteady behaviour when $\epsilon$ is small.

Substitution of the inner expansion

$$
\theta=\theta_{0}(z, t)+\epsilon \theta_{1}(z, t) \cdots
$$

into (41), with $\zeta(t)$ represented by (28), and replacement of $g(\theta)$ and $\kappa(\theta)$ by Taylor expansions gives the equations

$$
\begin{gathered}
\frac{\partial}{\partial Z}\left\{\kappa_{0} \frac{\partial \theta_{0}}{\partial Z}+\zeta^{(0)}(t) b_{0}+g_{0}\right\}=0, \\
\frac{\partial^{2}}{\partial Z^{2}}\left(\kappa_{0} \theta_{1}\right)+\frac{\partial}{\partial Z}\left(\beta_{0} \theta_{1}\right)=\frac{\partial b_{0}}{\partial t}-\zeta^{(1)^{\prime}} \frac{\partial b_{0}}{\partial Z},
\end{gathered}
$$

obeyed by the coefficients, where $b_{0} \equiv b\left(\theta_{0}\right)$, etc.

Eq. (43), (44), $\cdots$ indicate that hysteresis is present at all orders, first appearing in (43) through the coefficient $\kappa_{0}$. However, the problem remains tractable since the inner representation covers a region involving wetting only and for which the initial state in the hysteresis cycle is prescribed by the outer representation. This will become more evident with the matching of the inner and outer expansions. 
Evidently (43) and (44) can be integrated as quasi-ordinary differential equations in $z$, treating $t$ as a parameter. However, this situation does not hold when the diffusing front is within a distance of $0(\epsilon)$ from a boundary, such as the boundaries at $z=1, \xi(t)$. That case will be excluded from the present discussion.

Two integrations of (43) give successively

$$
\begin{aligned}
\kappa_{0} \frac{\partial \theta_{0}}{\partial Z} & =A_{0}(t)-\zeta^{(0)^{\prime}}(t) b_{0}-g_{0} \\
& =R\left(\theta_{0}, t\right) \equiv R_{0}, \text { say } \\
Z+B_{0}(t) & =\int^{\theta_{0}} \frac{\kappa(\theta) d \theta}{R(\theta, t)},
\end{aligned}
$$

where $A_{0}(t)$ and $B_{0}(t)$ are arbitrary functions.

Similarly, the results of integrating (44) twice are

$$
\begin{aligned}
\frac{\partial}{\partial Z}\left(\kappa_{0} \theta_{1}\right)+\beta_{0} \theta_{1} & =A_{1}(t)+\int_{0}^{Z} \frac{\partial b_{0}}{\partial t} d Z-\zeta^{(1) '}(t) b_{0} \\
& =S\left(\theta_{0}, t\right) \equiv S_{0}, \text { say } \\
\theta_{1}(Z, t) & =\frac{R_{0}}{\kappa_{0}}\left\{\int_{0}^{Z} \frac{S_{0} d Z}{R_{0}}+B_{1}(t)\right\}
\end{aligned}
$$

in which $A_{1}(t)$ and $B_{1}(t)$ are arbitrary functions. The lower limit in the integrals of $(46 \mathrm{a}, \mathrm{b})$ has been taken zero for convenience in relating to the outer expansion.

The determination of $A_{0}(t), B_{0}(t), A_{1}(t)$ and $B_{1}(t)$ involves both asymptotic matching and the application of the moisture conservation condition.

For the region close to the jump, write the outer expansion (11) in terms of inner variables as

$$
\begin{aligned}
\theta(z, t) & =\theta^{(0)}\{\zeta(t)+\epsilon Z, t\}+\epsilon \theta^{(1)}\{\zeta(t), t\}+\cdots \\
& =\theta_{i}{ }^{(0)}(t)+\epsilon\left\{\theta_{i}{ }^{(1)}(t)+Z\left(\partial \theta^{(0)} / \partial z\right)_{i}\right\}+\cdots
\end{aligned}
$$

for $i=1,2$. Then the matching conditions are

$$
\begin{aligned}
& \theta_{0}(Z, t) \rightarrow \theta_{i}{ }^{(0)}(t), \\
& \theta_{1}(Z, t) \rightarrow \theta_{i}{ }^{(1)}(t)+Z\left(\partial \theta^{(0)} / \partial z\right)_{i},
\end{aligned}
$$

where $Z \rightarrow-\infty,+\infty$ for $i=1,2$ respectively as $\epsilon \rightarrow 0$.

Since the conservation condition has already been satisfied in the determination of $\zeta(t)$, and the inner expansion about $\zeta(t)$ represents only a redistribution in the neighbourhood of the jump, it is necessary that

$$
\int_{-\infty}^{\infty}\left\{b_{(\text {inner })}-b_{\text {(outer) }}\right\} d Z=0
$$

If $b_{\text {(inner) }}$ and $b_{\text {(outer) }}$ are replaced by the expansions (42) and (47) respectively, (49) is equivalent to

$$
\int_{-\infty}^{\infty}\left\{b_{0}-b_{1}{ }^{(0)}(t)\right\} d Z=\int_{\theta_{1}(0)}^{\theta_{2}(0)} Z d b_{0}=0
$$




$$
\int_{-\infty}^{\infty}\left\{\theta_{1} b_{0}{ }^{\prime}-b_{i}{ }^{(0)}{ }^{\prime}\left(\theta_{i}{ }^{(1)}+Z\left(\partial \theta^{(0)} / \partial z\right)_{i}\right)\right\} d Z=0,
$$

where $i=1$ for $Z<0$ and $i=2$ for $Z>0$.

For the function $A_{0}(t)$, application of the matching conditions (48a) for $i=1,2$ to (45a), noting that $\partial \theta_{0} / \partial Z \rightarrow 0$ in each case, gives

$$
A_{0}(t)=\zeta^{(0) \prime}(t) b_{1}{ }^{(0)}(t)+g_{1}{ }^{(0)}(t)=\zeta^{(0) \prime}(t) b_{2}{ }^{(0)}(t)+g_{2}{ }^{(0)}(t) \text {. }
$$

The implicit relationship (51b) between $\theta_{1}{ }^{(0)}(t)$ and $\theta_{2}{ }^{(0)}(t)$ determines $\zeta^{(0)}{ }^{\prime}(t)$; in fact, the jump condition (31) has been recovered.

To determine $A_{1}(t)$, the matching conditions (48b) are applied to (46a), noting that $\partial \theta_{1} / \partial Z \rightarrow\left(\partial \theta^{(0)} / \partial z\right)_{i}$. If the subscript $(t)$ is dropped temporarily, $\kappa_{i}{ }^{(0)}\left(\partial \theta^{(0)} / \partial z\right)_{i}+\beta_{i}{ }^{(0)} \theta_{i}{ }^{(1)}+\zeta^{(1) '} b_{i}{ }^{(0)}-A_{1}$

$$
\begin{aligned}
& =\lim _{\substack{i \in 0 \\
|Z| \rightarrow \infty}}\left\{\int_{0}^{Z}\left(\partial b_{0} / \partial t\right) d Z-\beta_{i}{ }^{(0)}\left(\partial \theta^{(0)} / \partial z\right)_{i} Z\right\} \\
& =\lim \left\{\int_{0}^{Z}\left(\partial b_{0} / \partial t\right) d Z-\partial b_{i}{ }^{(0)} / \partial t Z\right\} \\
& =\lim \frac{\partial}{\partial t}\left\{\left(b_{0}-b_{i}{ }^{(0)}\right) Z-\int^{\theta_{0}} Z d b_{0}\right\}=-\frac{\partial}{\partial t} \int^{\theta_{1}(0)} Z d b_{0}
\end{aligned}
$$

for $i=1,2$, where the lower limits of the final two integrals correspond to $Z=0$. In (52), use has been made of the relation

$$
\partial b^{(0)} / \partial t=\beta^{(0)} \partial \theta^{(0)} / \partial z
$$

derived from (13), which applies at the moving discontinuity. Elimination of $A_{1}(t)$ from (52) gives

$$
\left[\kappa^{(0)} \partial \theta^{0} / \partial z\right]_{1}^{2}+\left[\beta^{(0)} \theta^{(1)}\right]_{1}{ }^{2}+\left[b^{(0)}\right]_{1}^{2} \zeta^{(1) \prime}=-\frac{\partial}{\partial t} \int_{\theta_{1}(0)}^{\theta_{2}(0)} Z d b_{0},
$$

and the right-hand side is zero by (50a). Thus the jump condition (32) has been recovered.

If $A_{1}(t)$ is now eliminated between (52) and (46a), it is found that

$$
\begin{aligned}
S_{0}= & \kappa_{i}{ }^{(0)}\left(\partial \theta^{(0)} / \partial z\right)_{i}+\beta_{i}{ }^{(0)}\left\{\theta_{i}{ }^{(1)}+Z\left(\partial \theta^{(0)} / \partial z\right)_{i}\right\} \\
& +\left(b_{i}{ }^{(0)}-b_{0}\right) \zeta^{(1)^{\prime}}+Z(\partial / \partial t)\left(b_{0}-b_{i}{ }^{(0)}\right)+\frac{\partial}{\partial t} \int_{\theta_{0}}^{\theta_{i}\left({ }^{(0)}\right.} Z d b_{0}
\end{aligned}
$$

for $i=1,2$.

At this stage, the inner expansion remains non-unique, since the matching requirements (48) have been satisfied without reference to the values of $B_{0}(t)$ and $B_{1}(t)$. The terms involving $B_{0}(t)$ (corresponding to a small translation of the $\theta_{0}$-profile) and $B_{1}(t)$ (which vanishes in the matching) behave as eigensolutions [26]. These functions are determined by the conservation conditions $(50 \mathrm{a}, \mathrm{b})$.

Substitution of (45b) for $Z$ in (50a) gives at once

$$
B_{0}(t)\left[b^{(0)}\right]_{1}{ }^{2}=\int_{b_{1}(0)}^{b_{2}(0)} d b_{0} \int^{\theta_{0}} \frac{\kappa(\theta) d \theta}{R(\theta, t)}
$$


so that

$$
Z\left[b^{(0)}\right]_{1}{ }^{2}=\int_{\theta_{1}(0)}^{\theta_{0}}\left\{b(\theta)-b_{1}{ }^{(0)}\right\} \frac{\kappa(\theta) d \theta}{R(\theta, t)}-\int_{\theta_{0}}^{\theta_{2}(0)}\left\{b_{2}{ }^{(0)}-b(\theta)\right\} \cdot \frac{\kappa(\theta) d \theta}{R(\theta, t)}
$$

An expression for $B_{1}(t)$ results from (46b) and (50b); viz.,

$$
-B_{1}(t)\left[b^{(0)}\right]_{1}{ }^{2}=\int_{-\infty}^{\infty}\left\{\frac{\partial b_{0}}{\partial Z} \int_{0}^{Z} \frac{S_{0} d Z}{R_{0}}-b_{i}{ }^{(0)}{ }^{\prime}\left[\theta_{i}{ }^{(1)}+Z\left(\partial \theta^{(0)} / \partial z\right)_{i}\right]\right\} d Z .
$$

Then, elimination of $B_{1}(t)$ between (58) and (46b) leads to

$$
\begin{aligned}
\theta_{1}\left[b^{(0)}\right]_{1}{ }^{2}= & {\left[b^{(0)}\right]_{1}{ }^{2} \frac{\partial \theta_{0}}{\partial Z} \int_{0}^{Z} \frac{S_{0} d Z}{R_{0}}-\frac{\partial \theta_{0}}{\partial Z} } \\
& \cdot \int_{-\infty}^{\infty}\left\{\frac{\partial b_{0}}{\partial Z} \int_{0}^{Z} \frac{S_{0} d Z}{R_{0}}-b_{i}{ }^{(0)}\left[\theta_{i}{ }^{(1)}+Z\left(\frac{\partial \theta^{(0)}}{\partial z}\right)_{i}\right]\right\} d Z .
\end{aligned}
$$

The right-hand side can also be expressed in terms of $\theta_{0}$ instead of $Z$.

Eqs. (57) and (59) give the coefficients of the first and second approximations (the former in inverse form) for the inner expansion (42).

An expansion uniformly valid for all $z$-i.e. including the ranges of both inner and outer expansions - is readily formed by adding the outer and inner expansions (11) and (42), and then subtracting the 'common part' (47) [27]; formally,

$$
\begin{aligned}
\theta(z, t)=\theta^{(0)}(z, t) & +\theta_{0}(Z, t)-\theta_{i}{ }^{(0)}(t) \\
& +\epsilon\left\{\theta^{(1)}(z, t)+\theta_{1}(Z, t)-\theta_{i}{ }^{(1)}(t)-Z\left(\partial \theta^{(0)} / \partial z\right)_{i}\right\}+\cdots,
\end{aligned}
$$

where $i=1,2$ for $z=\zeta(t)+\epsilon Z \lessgtr \zeta(t)$ respectively.

Asymptotic behavior. From the forms of the leading (dominant) terms it is readily verified that the inner expansion converges to the outer expansion exponentially rapidly when $\theta_{0} \rightarrow \theta_{i}{ }^{(0)}(i=1,2)$ for $Z \rightarrow \mp \infty$ respectively.

In (57), if $\theta_{0} \rightarrow \theta_{i}{ }^{(0)}$ with $i=1,2$, the dominant contributions arise from the second and first integrals respectively. Since

$$
R_{0} \sim-{\beta_{i}}^{(0)}\left(\theta_{0}-{\theta_{i}}^{(0)}\right)+0\left(\theta_{0}-{\theta_{i}}^{(0)}\right)^{2}
$$

from (7.2a) and (7.8), ti follows that

$$
\left|\theta_{0}-\theta_{i}{ }^{(0)}\right| \sim \exp \left(-W_{i}\right),
$$

writing $W_{i} \equiv W_{i}(Z, t) \equiv{\beta_{i}}^{(0)} Z / \kappa_{i}{ }^{(0)}>0$ since ${\beta_{i}}^{(0)} \lessgtr 0$ for $Z \rightarrow \mp \infty$.

The coefficient of $\exp \left(-W_{i}\right)$ in $(62)$ is $O\left(\theta_{2}{ }^{(0)}-\theta_{1}{ }^{(0)}\right)$. Its exact value, which depends upon the functions $\kappa(\theta)$ and $b(\theta)$, is not important for the present discussion.

With the aid of (62), Eq. (55) gives the asymptotic form

$$
S_{0} \sim \kappa_{i}{ }^{(0)}\left(\partial \theta^{(0)} / \partial z\right)_{i}+{\beta_{i}}^{(0)}\left\{\theta_{i}{ }^{(1)}+Z\left(\partial \theta^{(0)} / \partial z\right)_{i}\right\}+O\left(Z \exp \left[-W_{i}\right]\right)
$$

for $i=1,2$.

For the asymptotic form of (59), it is sufficient to observe, from (61), (62) and (63), that

$$
\frac{\partial \theta_{0}}{\partial Z} \int_{0}^{Z} \frac{S_{0} d Z}{R_{0}} \sim \theta_{i}^{(1)}+Z\left(\partial \theta^{(0)} / \partial z\right)_{i}+O\left(Z^{2} \exp \left[-W_{i}\right]\right)
$$


Then the integrand of the infinite integral in (59) vanishes exponentially at the limits; that is, the integral is convergent and $B_{1}(t)$ exists. The first term of (59) matches successfully to the outer expansion for $i=1,2$, as it should, since the matching was performed on (46a) after a single integration of the differential equation (44).

Inner expansion at downstream boundary.

For the "backward-facing" profile at the water-table $z=\xi(t)$, it is evident that a quasi-steady situation again exists, provided that the upstream value $\theta(\xi, t)$ (determined by the outer expansion) is slowly-varying. As before, the appropriate thickness scale is $\epsilon$, and a "stretched" variable $Z$ can be defined using (40) with $\zeta(t)$ replaced by $\xi(t)$. This replacement of $\zeta(t)$ is also made in equations (41) ff.

Expressions formally equivalent to (45), (46), $\cdots$ then follow, with arbitrary functions $A_{0}(t), B_{0}(t), A_{1}(t), B_{1}(t), \cdots$ to be determined. The problem differs mainly in the matching conditions; equations $(48 \mathrm{a}, \mathrm{b})$ become

$$
\begin{aligned}
& \theta_{0}(Z, t) \rightarrow \begin{cases}\theta^{(0)}(\xi, t) & (Z \rightarrow+\infty) \\
\theta_{\theta} & (Z \rightarrow-\infty)\end{cases} \\
& \theta_{1}(Z, t) \rightarrow \begin{cases}\theta^{(1)}(\xi, t)+Z \partial \theta^{(0)} /\left.\partial z\right|_{z=\xi(t)} & (Z \rightarrow+\infty) \\
0 & (Z \rightarrow-\infty)\end{cases}
\end{aligned}
$$

It is apparent that the water-table discontinuity is replaced by a smooth diffusionlike profile. The definition of the equivalent discontinuity by continuity requirements, as above, is convenient, and may coincide with a definition based on "capillary rise" which has appeared frequently in the literature.

Conclusion. In this paper, the approach to solving equation (7) has been dictated mainly by the expected high degree of nonlinearity in the coefficients $b(\theta), g(\theta)$ and $\kappa(\theta)$, and the arbitrary nature of the input flux $f(t)$ which may contain large-amplitude fluctuations. For solution by analytical means, there is some advantage if the system is hyperbolic, which is the case here when $\epsilon=0-$ a guide to the perturbation strategy actually adopted.

When $\epsilon \ll 1$, the physical properties of the system remain "quasi-hyperbolic", and large-amplitude waves in $f(t)$ will propagate with change of form. The values of the second derivatives of $b(\theta)$ and $g(\theta)$ are important, since the local wave velocity $-1 / a\left(\theta^{(0)}\right)$ increases in magnitude with $\theta$ provided that

$$
g^{(0) \prime \prime} / b^{(0) \prime}-g^{(0) \prime} b^{(0) \prime \prime} / b^{(0) \prime 2}>0
$$

In this case, the profile may be expected to steepen in the "forward-facing" direction, although the diffusive effect arising from capillarity prevents the actual formation of discontinuities. When the inequality in (65) is reversed, the steepening is "backwardfacing"- a situation which can arise in some problems of ion transport.

Observed attenuation of maxima in some experimental moisture profiles arises from the modification of wave shape with time due to non-linearity. In the situation considered here, significant attenuation will not occur until a pseudo-discontinuity has formed and has passed through a maximum value; the subsequent decay in amplitude is dependent upon the shape of the "tail" in the profile behind the jump. 
Capillarity has an important influence upon the shape of an advancing front, but the velocity of advance is modified (due to net diffusive transport into or out of the jump) to $0(\epsilon)$ only. For crests or troughs of the $\theta$-wave far from a jump, the influence of capillarity is $o(\epsilon) .\left(\theta^{(1)}=0\right.$ in (24).) This supports the above contention that wave attenuation is mainly a non-linear phenomenon when $\epsilon$ is small.

Capillary hysteresis influences the coefficient $\kappa(\theta)$ in equation (7). A change of dependent variable from $\theta$ to $\int \kappa(\theta) d \theta$ would "linearize" the diffusion term, but the new variable would itself be subject to hysteresis. Fortunately, capillarity does not have a major effect upon the flow at small $\epsilon$, so that approximations to $\kappa(\theta)$ are worth considering. Also, the perturbation method implies a basic simplification, since the gravity-induced flow, corresponding to the first term in the outer expansion, involves only parameters which are not significantly affected by hysteresis. Thus the first term serves to determine a first approximation to the previous mositure history, independent of hysteresis effects. In principle, the corresponding capillary potential functions can then be determined, either by means of a suitable theoretical hysteresis model or by simulation. In the case of the inner expansion, hysteresis appears at the first term, but the above argument still applies provided that rate-dependence is negligible.

Concerning the magnitude of the parameter $\epsilon$, it has been observed [23] that the range of $\alpha$ in (7b) is roughly 0.002 to 0.05 , with typical values of order 0.01 . For a sandy loam [17], $\alpha \approx 0.016$ on a typical scanning curve of the hysteresis diagram. For $\epsilon$ to be "small"-e.g. less than 0.3 -it is evidently necessary that $L$ be large, say 2 or 3 metres, and that the permeability should be fairly high, corresponding to the higher values of $\alpha$.

\section{Range of validity of the method.}

In the application of the perturbation method, the aim has been to provide expansions uniformly valid in space. Expansions uniformly valid in time have not been achieved. Instances of breakdown are (1) at the initial formation of a "head shock", (2) at the coalescence of two discontinuities, such as the arrival of the established "head shock" (discussed above) at the water-table, and (3) at the first appearance of a discontinuity at the inlet boundary $z=1$ due to a finite discontinuity in $f(t)$. In (1), the outer expansion breaks down, and in all three cases the quasi-stationary approximation, for which the inner expansion is valid, breaks down. In the latter situation, the $\partial^{2} / \partial z^{2}$ term dominates the $\partial / \partial z$ term, and is balanced by $\partial / \partial t$. Since the approximation regains validity after a time exceeding $0(\epsilon)$ has elapsed, the expansions are applicable for sufficiently large time.

A further special case can arise when the velocity function $g^{\prime}(\theta)$ vanishes as $\theta \rightarrow 0$. Suppose that $g^{\prime}(\theta) \sim \theta^{n}(n>0)$ and let $\theta$ be small. (The case $n=1$ corresponds to Burgers' equation [14].) Putting $\theta=\epsilon^{1 / n} \Theta$ in (7) gives

$$
\frac{\partial \Theta}{\partial t} \sim \epsilon\left\{\frac{\partial}{\partial z}\left(\kappa \frac{\partial \Theta}{\partial z}\right)+\Theta^{n} \frac{\partial \Theta}{\partial z}\right\}
$$

so that the first approximation is a stationary profile with $\Theta \equiv \Theta(z)$. This would be the appropriate condition ahead of a large-amplitude wave moving into a region of low $\theta$-values.

\section{APPENDIX}

The jump coordinate $z=\zeta(t)$ must satisfy the integral conservation condition

$$
\int_{\theta-\theta_{\mathbf{L}}(t)}^{\theta=\theta_{\mathbf{x}}(t)}\{z-\zeta(t)\} d b(\theta)=0
$$


where $\theta_{1}(t), \theta_{2}(t)$ represent values just ahead of, and just behind, the jump. For given $t, z$ is assumed to be a known function of $\theta$ through the asymptotic expansion (11). Although the values assumed by $z$ in the range of integration are not physically relevant, the solution satisfies formally the conservation condition.

Evaluation of (A1) is not straightforward, since (11) is not uniformly valid in the range of integration. However, the singularity in $\theta$ can be removed (at the expense of introducing a less troublesome singularity in $z$ ) by a coordinate perturbation analogous to Lighthill's method [28].

Let

$$
z=Z_{*}+\epsilon z^{(1)}\left(Z_{*}, t\right)+\cdots
$$

define a new coordinate $Z_{*}$. Substituting in (11) gives

$$
\theta(z, t)=\theta^{(0)}\left(Z_{*}, t\right)+\epsilon\left\{z^{(1)}\left(Z_{*}, t\right) \frac{\partial \theta^{(0)}}{\partial z}\left(Z_{*}, t\right)+\theta^{(1)}\left(Z_{*}, t\right)\right\}+\cdots
$$

after expansion by Taylor series. Now, if $z^{(1)}$ is defined so that the coefficient of $\epsilon$ in (A3) vanishes identically, the integral (A1) can be rewritten to give

$$
\begin{gathered}
\int_{1}^{2}\left\{Z_{*}-\zeta^{(0)}(t)\right\} d b^{(0)}=0 \\
\int_{1}^{2}\left\{\theta^{(1)} \Delta / a^{(0)}+\zeta^{(1)}(t)\right\} d b^{(0)}=0
\end{gathered}
$$

The relationship between $Z_{*}$ and $\theta^{(0)}$ is given by (20) (with $Z_{*}$ replacing $z$ ). With (A4), the result (36) follows. Also, (37) is immediately obtained from (A5) and (38).

\section{REFERENCES}

[1] J. R. Philip, Theory of infiltration, Adv. Hydrosci. 5, 215-296 (1969)

[2] C. Braester, G. Dagan, S. Neuman and D. Zaslavsky, A survey of the equations and solutions of unsaturated flow in porous media, 1st Ann. Rep. (Part 1), Project No. A10-SWC-77, TechnionIsrael Institute of Technology, 1971

[3] A. Poulovassilis and E. C. Childs, The hysteresis of pore water: The non-independence of domains, Soil Sci. 112, 301-312 (1971)

[4] G. C. Topp, Soil-water hysteresis: The domain theory extended to pore interaction conditions, Soil Sci. Soc. Amer. Proc. 35, 219-225 (1971)

[5] G. A. Turner, The flow-structure in packed beds, Chem. Eng. Sci. 7, 156-165 (1958)

[6] D. E. Hill and J. -Y. Parlange, Wetting front instability in layered soils, Soi Sci. Soc. Amer. Proc. 36, 697-702 (1972)

[7] H. J. Morel-Seytoux, Introduction to flow of immiscible liquids in porous media, 'Flow Through Porous Media', ed. by R. J. M. De Wiest Academic Press, New York, 455-516 (1969)

[8] A. J. Peck, Moisture profile development and air compression during water uptake by bounded porous bodies. 3. Vertical columns, Soil Sci. 100, 44-51 (1965)

[9] J. -Y. Parlange, Theory of water-movement in soils: I. One-dimensional absorption, Soil Sci. 111, 134-137 (1971)

[10] J. -Y. Parlange, Theory of water-movement in soils: II. One-dimensional infiltration, Soil Sci. 111, $170-174(1971)$

[11] A. Noblanc and H. J. Morel-Seytoux, Perturbation analysis of two-phase infiltration, J. Hydraul. Div. Proc. ASCE, 98 (HY9), 127-1541 (1972)

[12] R. A. Wooding, Perturbation analysis of the equation for the transport of dissolved solids through porous media. II. Basic non-linear problem. J. Hydrol. (Amsterdam) 16, 105-116 
[13] M. J. Lighthill and G. B. Whitham, On kinematic waves. I. Flood movement in long rivers, Proc. Roy. Soc. A 229, 281-316 (1955)

[14] M. J. Lighthill, Viscosity effects in sound waves of finite amplitude, 'Surveys in Mechanics' eds. G. K. Batchelor and R. M. Davies, University Press, Cambridge, 250-351 (1956)

[16] G. C. Topp and E. E. Miller, Hysteretic moisture characteristics and hydraulic conductivities for glass-bead media, Soil Sci. Soc. Amer. Proc. 30, 156-162 (1966)

[17] G. C. Topp, Soil-water hysteresis measured in a sandy loam and compared with the hysteretic domain model, Soil Sci. Soc. Amer. Proc. 33, 645-651 (1969)

[18] G. C. Topp, Soil water hysteresis in silt loam and clay loam soils, Water Resources Res. 7, 914-920 (1971)

[19] J. A. Enderby, The domain model of hysteresis. I. Independent domains, Trans. Faraday Soc. 51, 835-848 (1955)

[20] D. H. Everett, $A$ general approach to hysteresis. 4. An alternative formulation of the domain model, Trans. Faraday Soc. 51, 1551-1557 (1955)

[21] D. H. Everett, Adsorption hysteresis, 'Solid Gas Interface' ed. E. Alison Flood, 2, 1055-1113 (1967)

[22] W. R. Gardner, Some steady-state solutions of the unsaturated moisture fiow equation with application to evaporation from a water table, Soil Science, 85, 244-249 (1958)

[23] J. R. Philip, Steady infiltration from buried point sources and spherical cavities, Water Resources Res. 4, 1039-1047 (1968)

[24] R. A. Wooding, Steady infiltration from a shallow circular pond, Water Resources Res. 4, 12591273 (1968)

[25] R. A. Wooding, Perturbation analysis of the equation for the transport of dissolved solids through porous media. I. Linear problems, J. Hydrol. (Amsterdam) 16, 1-16 (1972)

[26] M. Van Dyke, Perturbation methods in fluid mechanics, Academic Press, New York, 1964

[27] J. D. Cole, Perturbation methods in applied mathematics. Blaisdell, Waltham, Mass., 1968

[28] L. Crocco, Coordinate perturbation and multiple scale in gas-dynamics, Phil. Trans. Roy. Soc. A. 272, 275-301 (1972) 